\title{
Intelligent City Lighting Supported by Graph-based Multiagent System
}

\section{Introduction}

The growing costs of energy and environmental care stimulate the development of technologies reducing the energy usage, efficient storage, its sustainable consumption and so on [11]. The area of potential benefits is an intelligent control of energy usage (see [2]). The intelligence is understood here as the ability to decision making based on a problemspecific knowledge. That knowledge may be compound of long-term data like an underlying environment structure and volatile knowledge about an actual environment state (e.g.from sensors).

In the paper we present the adaptive, predictive, agent-based lighting system for urban spaces, being the example of such an intelligent control. Moreover, the hierarchical metamodel of a system representation is introduced. Its properties make it applicable for distributed, agent-based computations.

The main objective of the proposed approach is satisfying all requirements implied by existing norms and regulations concerning lighting conditions, a place-specific architectural demands or other non formalized limitations and, on the other hand, minimizing power consumption (impacting exploitation costs) in compliance with these constraints. The problem being formulated above consists of two loosely related subproblems. The first, static one, is finding an optimal distribution of luminaries and their parameters in such a way that the exploitation cost is minimal. The second (dynamic) subproblem is adjusting a system performance to an actual environment needs. Note that both subproblems are constrained by existing lighting standards.

It should be emphasized that both subproblems have high computational complexities implied by a number of degrees of freedom (in particular related to a number parameters describing a luminary), size of the environment state space and so on. Such circumstances

* AGH University of Science and Technology, Department of Applied Computer Science, Krakow, Poland 
enforce using suitable computational methods, especially in the context of reactive control system.

Our approach to this problem is twofold. First we prepare the convenient formal model including the hierarchical data metamodel and suitable data structures. Next, the corresponding computational approach is proposed. In the sequel we will focus on the hierarchical data metamodel and intelligent control problem only.

The paper is organized as follows. In Section 2 we overview selected works related to intelligent lighting control. Section 3 describes the structural characteristics of systems being concerned. In Section 4 we introduce the hierarchical metamodel of an environment. The concept of lighting profiles is depicted in Section 5. Section 6 describes the architecture of a multiagent system managing the intelligent predictive lighting. The case study is presented in Section 7 and Section 8 summarizes the paper.

\section{Related works}

Advanced lighting technologies like LED (light-emitting diodes) or frequency controlled dimmable ballasts enable the stepless luminary control and thus open up the possibility of creating fine adjustable lighting systems, responding precisely to actual lighting needs of an environment. For this reason the related research effort focuses on effective algorithmic methods allowing to benefit the technological assets.

Functionality of existing industry solutions are limited to the preset dimming scenarios or switching scheduling rather than capable of making control decisions dependent on a varying environment state. Although the solution infrastructure contains all elements required to perform such actions: in particular, each individual lamp may controlled separately (e.g. Echelon's Smart Street Lighting).

The concept of the predictive lighting systems was investigated in the context of indoor lighting [8]. The proposed daylight-responsive lighting and shading systems control is semiautomatic in the sense that in some circumstances the human interaction is assumed. Interior lighting was discussed also in [10] as a part of the broader solution, controlling appliances on the basis of monitored parameters e.g. temperature, humidity, human motion and so on. The goal was the reduction of an exploitation cost. Another prototype implementation of an indoor lighting system, installed in an office space, is presented in [5]. The solution complexity may be assessed by the number of used components: 26 lighting fixtures and 22 illuminance sensors. In the paper [1] authors discuss ensuring required illuminance by adjusting both luminous intensity and height of a luminaire. In this case we deal with lighting design approach rather than with lighting control. Yet another work [7] describing an office space lighting, focuses on hybrid (supporting continuous and discrete parameters) platform for a building control. The presented control model is supported by a multiagent system. In particular there exists the special type of agent which models a person's movements. It is accomplished using stochastic methods, precisely Markov 
chain, to model the person presence in given areas ${ }^{*}$. The papers listed above are closely related to the BMS (Building Management System) concept. The specific outdoor lighting problem is automatic control system for highway tunnel LED lighting, presented in [4]. Entire tunnel space which has to be illuminated in accordance with compulsory regulations is subdivided functionally into five sections. Each of them has the separate illumination specification. Among the system's objectives is sewing those separate illuminance distributions at sections' endpoints and to ensure smooth transitions between different lighting level states. Due to a tunnel's translational symmetry (in the geometric sense) the problem of its illumination belongs to the simpler ones from the both algorithmic and system-architecture perspective.

\section{Environment description}

The preliminary assumption about an urban space is that it may be logically decomposed into basic types of entities: buildings, infrastructure components, pedestrian/car traffic areas (streets, pavements), non-traffic areas (buildings underlying grounds, lawns) and so on. The relevant data are accessible from a CityGML representation [6], for example, which enables switching easily among different granularities of a system description.

All those entities obtained in a result of logical decomposition are modeled by means of graphs/hypergraphs (see [9]).

a)

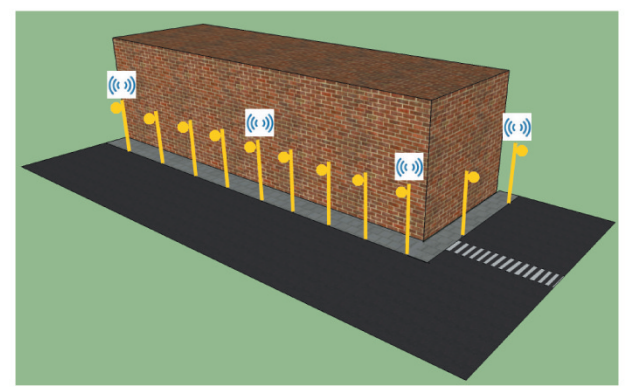

b)

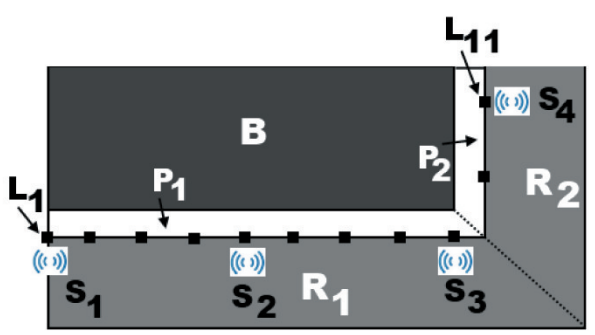

Fig. 1. Urban space environment. Luminaries and sensors are the assumed elements of an infrastructure (a). Orthogonal projection (b) of the scene shown in (a)

Figure 1(a) presents the typical scene being considered. It consists of sidewalks, roads and buildings. The only areas permitted for pedestrians are sidewalks and pedestrian crossings (in a general case it may be extended by other zones like lawns). Besides the above the area contains also infrastructural objects: luminaries and sensors. To simplify the case

* Tracing agent being another approach to the presence analysis is introduced in this paper (see Section 6). 
we assume without loss of generality that existing sensors are attached permanently to lighting poles. To obtain a formal model of such an environment we have to make two steps. In the first one we identify all existing zones and objects in the scene. The sample fragmentation made for the considered case is shown in Figure 1(b). The following notation is used: $B$ stands for the building object, $P$ stands for pavements, $R$ denotes a road, $L, S$ stand for luminaries and sensors respectively (the pedestrian crossings was neglected here).

There are possible various graph representations of an urban space [6,9]. To simplify the considered case we use the following one (see Fig. 2): regions obtained in a result of a scene fragmentation and an infrastructure elements like luminaries, sensors, are represented by a graph vertices (vertices related to zones are marked with circles, nodes corresponding to an infrastructure elements, with rectangles). Graph edges represent the relation of adjacency (between regions) and relation of including (between an infrastructure element and a region). For the scene being analyzed we obtain $G=(V, E, \Phi)$ where $V=\left\{P_{1}, P_{2}\right.$, $\left.R_{1}, R_{2}, B, L_{1}, \ldots, L_{11}, S_{1}, \ldots, S_{4}\right\}, E=\left\{\left\{B, P_{1}\right\},\left\{B, P_{2}\right\},\left\{R_{1}, P_{1}\right\},\left\{R_{2}, P_{2}\right\},\left\{R_{1}, R_{2}\right\}\right.$, $\left\{P_{1}, P_{2}\right\},\left\{S_{4}, R_{2}\right\},\left\{P_{2}, S_{4}\right\}\left\{L_{i}, R_{1}\right\}_{i=1, \ldots, 9},\left\{P_{1}, L_{i}\right\}_{i=1, \ldots, 9},\left\{S_{i}, R_{1}\right\}_{i=1,2,3},\left\{P_{1}, S_{i}\right\}_{i=1,2,3}$, $\left.\left\{L_{i}, R_{2}\right\}_{i=10,11}, \quad\left\{P_{2}, L_{i}\right\}_{i=10,11}\right\}$ and $\Phi$ is the set of labeling and attributing functions for vertices and edges.

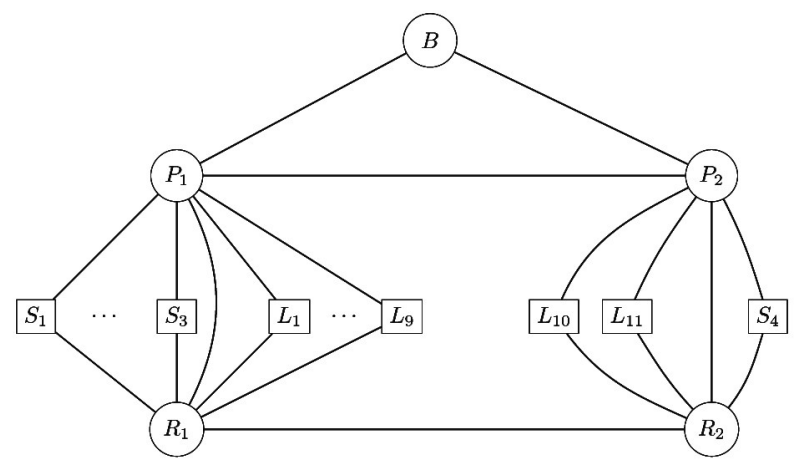

Fig. 2. The sample graph representation for the scene shown in Figure 1

The graph representing the area is obtained by the scene decomposition made on the basis of the above identification and supplementary criteria. Such criteria may be the functional ones, e.g. they may be implied by grouping luminaries located in a given zone.

Note that in the example discussed above we neglect geometric properties of objects. It is assumed implicitly that all those data are passed to a graph structure by means of attributing functions belonging to the $\Phi$ set.

\section{Hierarchical metamodel of environment representation}

This section depicts the hierarchical metamodel describing the computational structure of an urban space representation (see Fig. 3). 
The graph structure of an urban space representation, defined above, determines the top level of the hierarchy.

Due to the computational complexity implied by size of a graph $G$ corresponding to an entire area, it has to be decomposed into an atlas $\mathcal{A}$ being a set of subgraphs such that:

$$
\mathcal{A} \stackrel{\text { def }}{=}\left\{G_{i} \not \notin G: i \in I \text { and } \bigcup_{i \in I} G_{i}=G\right\}
$$

where $I$ is a finite set of indices. The lighting control tasks are processed independently on each element of $G_{i} \in \mathcal{A}$. Subgraphs $G_{i}$ constitute the 2nd level of the metamodel hierarchy.

A typical scene represented by $G_{i}$ contains zones obtained in a result of some arbitrary decomposition as it was discussed in Section 3. Obviously such a zone is also represented by a graph $G_{i(k)} \subseteq G_{i}, k \in K$, where $K$ is a finite set of indices. Subgraphs $\left\{G_{i(k)}\right\}_{k \in K}$ belong to the 3 rd level of metamodel hierarchy. Unlike at the 2nd level we have $\bigcup_{k \in K} G_{i(k)} \subseteq G_{i}$. The equality may be broken by nodes representing buildings and incident edges.

Objects belonging to the fourth (bottom) hierarchy level carry detailed geometric information related to underlying pieces of a scene. Such data may be obtained from values of a node attributing function $f \in \Phi$ (see Section 3) either in the form of a plain 2D geometric characteristics (e.g. as polygons) or some more sophisticated structure like a cellular automaton [12].

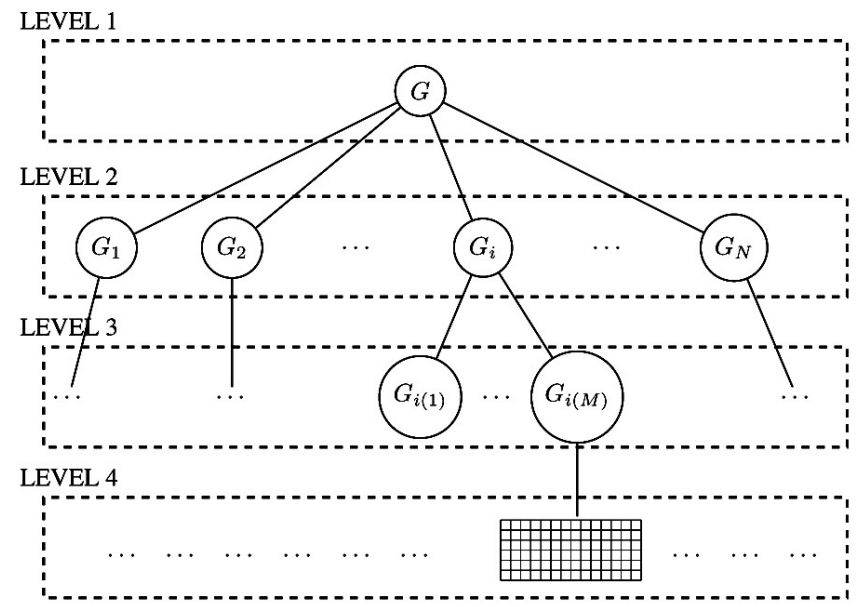

Fig. 3. The representation structure metamodel. Level 1 - main graph, level 2 - atlas, level 3 - zone subgraph, level 4 - zone 2D model

The important assumption about a bottom level object $a$ is that it may be obtained unambiguously from from a $G_{i(k)}$ graph and vice versa: $G_{i(k)}$ may be reconstructed from $a$. More formally, one can say that there exists bijection $f:$ LEVEL3 $\rightarrow$ LEVEL4. 
The question may arise, if is it possible to use bottom level representation straightforwardly instead of a whole structure. The following example shows that such an approach may be ineffective. Let us assume that we need to make calculations for a sidewalk sized $2 \mathrm{~m} \times 500 \mathrm{~m}$, using a cellular automaton with a cell size $0.3 \mathrm{~m} \times 0.3 \mathrm{~m}$. Then we obtain over 11,000 cells for a sidewalk representing automaton. This example demonstrates that the decomposition of a main problem is necessary to perform computations with the reasonable complexity.

\section{Lighting profiles and data input}

As it was mentioned in Section 1 the lighting control is partially determined by existing lighting standards (e.g. EN-13201 standards for roads) which provide a comprehensive specification of lighting conditions which has to be met, and which depends on a road type, weather conditions, vehicle speed and so on. Thus for a given area (e.g. street) we obtain a set of multiple lighting profiles, among which only one is active in a given moment, dependently on an actual environment state (see Fig. 4).
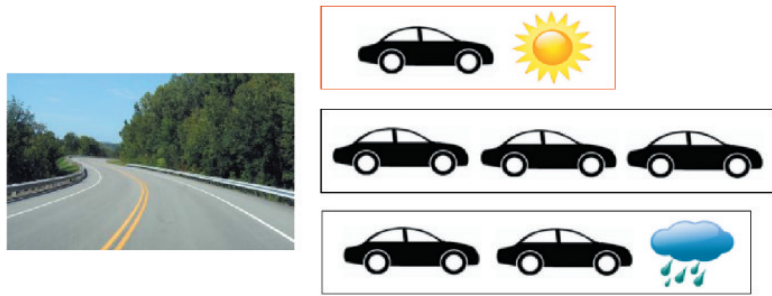

Fig. 4. Lighting profiles determined by a mutable environment state.

Only one profile is active in a moment

Lighting profiles play the important role in the intelligent lighting by supplying reference data for a control system. Another, crucial element of a lighting system are sensors providing information concerning an actual environment state. It's assumed that we have at least two types of devices: light level sensors and movement sensors which are capable of gathering coordinates of moving objects, $\mathbf{r}$, and thus determining also their velocity vectors $\mathbf{v}$.

All geometric constraints imposed on objects' movement (i.e. walls, streets or other obstacles) are stored in a graph structure mentioned in Section 4.

\section{Lighting system architecture}

The intelligent lighting control system is supported by a multiagent system containing the following types of agents. 
Operational agent (OA) resides at the 2nd level of the metamodel (Fig. 3); it is ascribed to a given portion of a graph representing an urban space. An operational agent has a knowledge about structural features of a corresponding area (e.g. coordinates of junctions or locations of buildings) but also about semantic features like a road type. An OA has two basic tasks: if no moving object (e.g. pedestrian or car) is present in an OA's maintained area then it controls a luminance within this space only by sending appropriate requests to particular luminary agents. Otherwise it creates a tracing agent bound to this object and delegates performing control tasks to this newly created agent.

Note that entire urban space is divided among operational agents.

Luminary agent (LA) lives at the 3rd level of the metamodel hierarchy, ascribed statically to a set of luminaries and performing direct control over their drivers. Its primary task is to optimize the performance of lamps against the actual environment state in compliance with a given lighting profile. An LA may accomplish that e.g. using the reasoning methods: for a given sensory data pattern and the current lighting profile the convenient control scheme is selected. It also smooths, in a cooperation with tracing agents (see Section 7), a work of luminaries to avoid frequent changes of luminosity levels. Another task of a luminary agent is reporting any technical failures to an operational agent.

Sensor agent (SA) is ascribed to a sensor device, supplying captured sensory data. Similarly as an LA, a sensor agent lives at the 3rd level of the metamodel hierarchy.

Tracing agent (TA) is ascribed to a moving object. A tracing agent is a volatile entity, which is created as soon as a new object appears on a scene and gets destroyed when an object leaves a considered area. TA receives a relevant piece of a graph (in the read-only mode) from an operational agent and transform it into some 2D representation (belonging to the 4th level of the hierarchy) being its calculation layer. Then for object's parameters $(\mathbf{r}, \mathbf{v})$ received from a sensor agent, TA determines both an object's current and predicted coordinates. As soon as both parameters are known, a tracing agent requests corresponding LAs to adjust luminosity levels correspondingly (see Section 7).

Since a tracing agent may be relayed among neighboring subgraphs of the 2 nd level it populates both the 1 st and the 2 nd level of the hierarchy. It should be remarked that existence of a bijection between TA owned piece of graph and 2D representation of this piece guarantees that any TA's predictions may be returned to an OA in the form of a graph.

An operational agent maintains its domain defined by a part of a graph representing an urban area (Fig. 5). In his domain exists a number of sensor agents and luminary agents which are stationary i.e. they are bound permanently to existing devices. A sensor agent delivers captured data to an operational agent using the unidirectional communication: an OA receives sensory data only, no requests to a SA are sent. An operational agent communicates with luminary agents using the bidirectional communication: it sends requests concerning a luminary performance adjustments and receives info e.g. about a luminary actual state. A tracing agent is either created by an operational agent or may relayed to it by one of the adjacent operational agents when the corresponding object leaves its domain. If an objects leaves a system then its tracing agent is destroyed. Besides the bidirectional 
communication with a master operational agent, a tracing agent sends requests to luminary agents via an unidirectional communication channel and receives sensor data from a sensor agent (also unidirectionally).

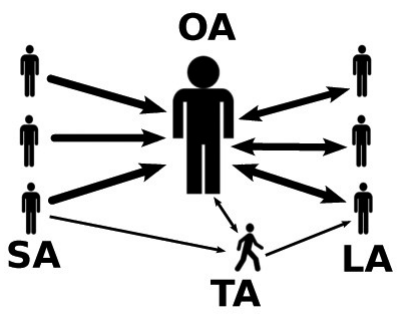

Fig. 5. Multiagent system architecture

\section{Case study. Prediction capability}

Let us consider the scene being discussed in Section 3. The scene (see Fig.6(a)) consists of the sidewalk decomposed logically into zones $P_{1}, P_{2}$, road decomposed analogously into zones $R_{1}, R_{2}$ and the building $B$. Luminaries $L_{1}, \ldots, L_{9}$ are identical and situated on the border between $P_{1}$ and $R_{1}$. A distance between lighting poles is 30.5 meters and a pole height is 4.6 meters. Analogously, $L_{10}, L_{11}$ are adjacent to $P_{2}$ and $R_{2}$, a distance between those lamps is 45.7 meters and poles' height is 7.6 meters $^{* *}$. The sidewalk width is 4 meters. The origin is associated with a base of a luminary $L_{1}$. In the following example we analyze a movement of two independent pedestrians denoted as $\mathrm{X}$ and $\mathrm{Y}$.

a)

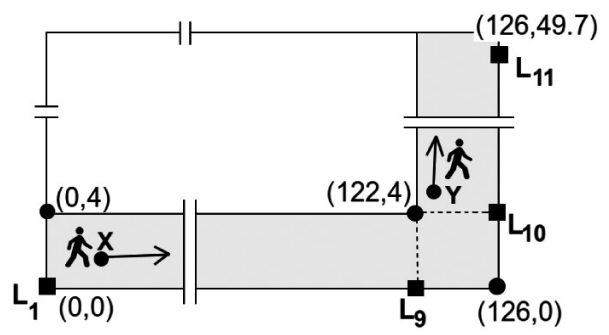

b)

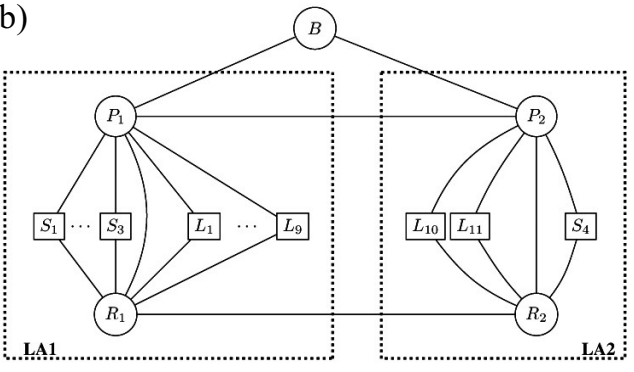

Fig. 6. The part of the scene with characteristic points' coordinates and pedestrians $X$ and $Y$ (a). The graph of the complete scene with dotted lines bordering the fragments managed by luminary agents LA1 and LA2 (b)

** The values are taken from the British Recommendation For The Lighting Of Traffic Routes (Group “A”) [3]. 
We assume that both pedestrians move in directions pointed by the velocity vectors and they are "shadowed" by tracing agents TAX and TAY respectively.

There are two luminary agents in the system as well: LA1 is responsible for the control of luminaries $L_{1}, \ldots, L_{9}$ and LA2 responsible for $L_{10}, L_{11}$ (see Fig. 6(b)). Sensor agents SA1, .., SA4 are associated with sensors $S_{1}, \ldots, S_{4}$.

To track changes in the luminance level along a pedestrian's path we work with the simplified model of a fixture. It is assumed that a fixture produces the isotropic light (i.e. spherically symmetric). Additionally, its control scheme is dependent on a sensory-detected presence of an object and is given by the following intensity function:

$$
I(d)=\frac{c(d)}{d^{2}}, \quad \text { where } \quad c(d)= \begin{cases}p & \text { when } d \leq R \\ 0.2 p & \text { when } d>R\end{cases}
$$

$d$ denotes a distance between a fixture and an object, $R$ is a cutoff radius and $c$ is the variable coefficient (corresponding to the fixture power). In general the form of $c(d)$ function depends on a control strategy.

The exemplary LA1's control scheme for the luminary $L_{i}$ may be depicted as follows. Having a pedestrian position provided by sensor agents, LA1 may determine its distance $d_{i}$ to the luminary $L_{i}$. In our simplified model (see eq. (1)) it is only required to test $d_{i}$ against the cutoff radius $R$, and to select the proper form of $c(d)$ coefficient. The expression (1) means that a luminary works in a minimal power mode ( $20 \%$ of a normal power level) when no object is present within a neighborhood of a radius $R$.

When assumed that a fixture is located in a point $\mathbf{q}$ then the luminosity in point $\mathbf{r}$ may be computed from (1) as:

$$
I(\mathbf{r})=\frac{c(\mathbf{r})}{|\mathbf{q}-\mathbf{r}|^{2}}, \quad \text { where } \quad c(\mathbf{r})= \begin{cases}p & \text { when }|\mathbf{q}-\mathbf{r}| \leq R \\ 0.2 p & \text { when }|\mathbf{q}-\mathbf{r}|>R\end{cases}
$$

We start the trial in a moment $t_{0}=0$ when X enters the area covered by the sensor $S_{2}$. In this moment TAX receives actual $X$ 's coordinates $\left(\mathbf{r}_{X, 0}\right)$ and velocity $\left(\mathbf{v}_{X}\right)$ from SA2. Analogously, TAY receives vectors $\mathbf{r}_{Y, 0}$ and $\mathbf{v}_{Y}$ from SA4, describing position and velocity of the pedestrian Y. Assuming that $\mathbf{v}_{X}$ and $\mathbf{v}_{Y}$ are constant in the time a pedestrian trajectory is:

$$
\mathbf{r}_{\mu}(t)=\mathbf{r}_{\mu, 0}+\mathbf{v}_{\mu} t, \quad \mu=X, Y
$$

By the equations (2) and (3) we obtain a luminance on a pedestrian's trajectory, parameterized by time:

$$
I(t)=\frac{c_{\mu}(t)}{\left|\mathbf{q}-\left(\mathbf{r}_{\mu, 0}+\mathbf{v}_{\mu} t\right)\right|^{2}}, \quad \mu=X, Y
$$


where

$$
c_{\mu}(t)= \begin{cases}p & \text { when }\left|\mathbf{q}-\left(\mathbf{r}_{\mu, 0}+\mathbf{v}_{\mu} t\right)\right| \leq R \\ 0.2 p & \text { when }\left|\mathbf{q}-\left(\mathbf{r}_{\mu, 0}+\mathbf{v}_{\mu} t\right)\right|>R\end{cases}
$$

To obtain a total luminance generated by entire assembly of fixtures, in a trajectory point, parametrize by time, one has to sum (4) over that set of fixtures:

$$
I_{t o t}(t)=\sum_{i} I_{i}(t)=\sum_{i} \frac{c_{i, \mu}(t)}{\left|\mathbf{q}-\left(\mathbf{r}_{\mu, 0}+\mathbf{v}_{\mu} t\right)\right|^{2}}
$$

Both tracing agents send requests to respectively LA1 and LA2 to update luminaries' work parameters according to pedestrians' movement characteristics. Precisely, it concerns $c\left(\mathbf{r}_{\mu}\right),(\mu=X, Y)$ functions (see eq. (2)).

The points where a pedestrians leave their zones may be easily calculated as an intersection of a trajectory and a zone border. Let us denote them as $\mathbf{r}_{X, f}$ and $\mathbf{r}_{Y, f}$ respectively. Then we obtain from (3) the time a pedestrian has to leave a zone:

$$
\Delta t_{\mu}=\frac{\left|\mathbf{r}_{\mu, f}-\mathbf{r}_{\mu, 0}\right|}{\left|\mathbf{v}_{\mu}\right|}, \quad \mu=X, Y
$$

After determining $\Delta t_{X}$ TAX requests LA2 to adjust working parameters of its luminaries when the pedestrian $\mathrm{X}$ reaches the zone border. Note that this request becomes meaningful when the considered LA handles more than one pedestrian. In such circumstances the received prediction allows avoiding short-time power switchings.

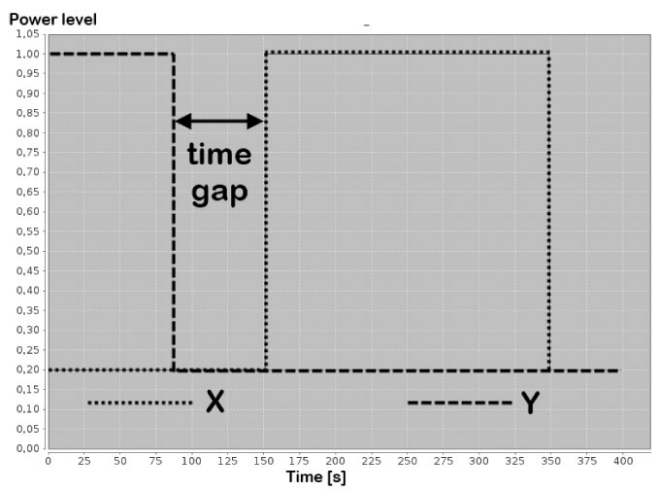

Fig. 7. The gap in the power level for luminary $L_{10}$ related do a movement of pedestrians $\mathrm{X}$ and $\mathrm{Y}$ 
Let us put following initial positions (in $|m|$ ) of pedestrians and their velocities (in $[\mathrm{m} / \mathrm{s}]): \mathbf{r}_{X, 0}=(0.5,2.0,0), \mathbf{r}_{Y, 0}=(125.0,10.0,0), \mathbf{v}_{X}=(0.5,1 / 300,0) ; \mathbf{v}_{Y}=(1 / 300,0.5,0)$. For those values we determine curves $C_{10, X}(t)$ and $C_{10, Y}(t)$ calculated for luminary $L_{10}$ (Fig. 7). The plot shows that $L_{10}$ power level switches to the low mode (when Y shifts beyond the cutoff area) and back again to the normal one (when X gets into the cutoff area). The system task is to fill such gaps by predicting their occurrences and prolonging dynamically a luminary performance at a normal power level, if needed. For better clarity the presented case was extremely simplified. In practice, however, we deal with multiple adjacent zones and moving objects, varying external factors and lighting profiles as well.

\section{Conclusions}

The control of a city lighting performance is a task of the high computational complexity. It's constrained by three major factors: existing lighting standards, energy consumption and demand of a system's smooth behavior. To satisfy all those requirements one has to use formally defined, graph-based data structures enabling distributed, parallel processing. Gathering an environment state data and exploiting prediction mechanisms allow a near-future extrapolation of a system state and thus achieving an effect of a performance smoothing. The discussed approach is to be applied in the intelligent lighting system being a part of the smart grid solution, Green AGH Campus.

\section{References}

[1] Akita M., Miki M., Hiroyasu T., Yoshimi M., Optimization of the height of heightadjustable luminaire for intelligent lighting system. [in:] Proceedings of the 10th international conference on Artifical intelligence and soft computing: Part II, ICAISC'10, Berlin, Heidelberg, 2010. Springer-Verlag, pp. 355-362

[2] Behrooz F., Ramli A.R., Samsudin K., A survey on applying different control methods approach in building automation systems to obtain more energy efficiency. Vol. 6, Academic Journals, 2011, pp. $2308-2314$

[3] Bicknell C.R., Street lighting. recommendations for the lighting of traffic routes (group "A"), 2012. http://www.simoncornwell.com/lighting/ publications/journals/pl36/cb1.htm.

[4] Shijuan Fan, Chao Yang, and Zhiwei Wang. Automatic control system for highway tunnel lighting. [in:] Daoliang Li, Yande Liu, and Yingyi Chen (Eds), Computer and Computing Technologies in Agriculture IV, volume 347 of IFIP Advances in Information and Communication Technology. Springer, Boston 2011, pp. 116-123.

[5] Kaku F., Miki M., Hiroyasu T., Yoshimi M., Tanaka S., Nishida T., Kida N., Akita M., Tanisawa J., Nishimoto T., Construction of intelligent lighting system providing desired illuminance distributions in actual office environment. [in:] Proceedings of the 10th international conference on Artifical intelligence and soft computing: Part II, ICAISC'10, Berlin, Heidelberg, 2010. Springer-Verlag, pp. 451-460.

[6] Kolbe Th.H., Gröger G., Plümer L., CityGML - interoperable access to $3 D$ city models. [in:] Proceedings of the first International Symposium on Geo- Information for Disaster Management. Springer Verlag, 2005, pp. 21-23. 
[7] A. El-Din Mady, Boubekeur M., Provan G., Ryan C., Brown K.N., Intelligent hybrid control model for lighting systems using constraint-based optimisation. [in:] Emilio Corchado, Paulo Novais, Cesar Analide, Javier Sedano (Eds), SOCO, volume 73 of AISC, Springer, 2010, pp. 249259.

[8] Mahdavi A., Predictive simulation-based lighting and shading systems control in buildings. [in:] Building Simulation, vol. 1, Tsinghua University Press, copublished with Springer-Verlag GmbH, 2008, pp. 25-35.

[9] Sędziwy A., Representation of objects in agent-based lighting design problem. [in:] W. et al. Zamojski (Eds), Complex Systems and Dependability, vol. 170 of AISC. Springer Berlin - Heidelberg, 2012, pp. 209-223.

[10] Silva L.C., Dewana T., Iskandar P.M., G. Amal Punchihewa, Multiple sensor based autonomous monitoring and control for energy efficiency. [in:] Prahlad Vadakkepat and et al. (Eds), Trends in Intelligent Robotics, vol. 103 of Communications in Computer and Information Science, Springer Berlin Heidelberg, 2010, pp. 361-368.

[11] Sioshansi F. (Ed.), Smart Grid. Integrating Renewable, Distributed \& Efficient Energy. Academic Press, 2011.

[12] Wąs J., Gudowski B., Matuszyk P., New cellular automata model of pedestrian representation. [in:] El Yacoubi et al. (Eds), Cellular Automata, vol. 4173 of LNCS, Springer Berlin / Heidelberg, 2006, pp. 724-727. 